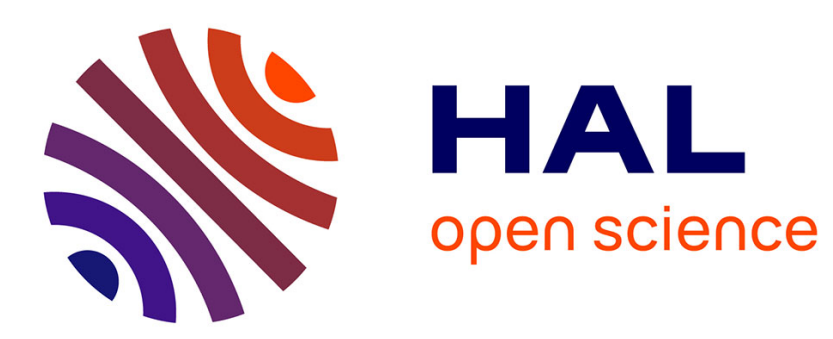

\title{
Cluster Poisson varieties at infinity
}

Vladimir V. Fock, Alexander Goncharov

\section{To cite this version:}

Vladimir V. Fock, Alexander Goncharov. Cluster Poisson varieties at infinity. Selecta Mathematica (New Series), 2016, 22 (4), pp.2569 - 2589. 10.1007/s00029-016-0282-6 . hal-01897341

\section{HAL Id: hal-01897341 \\ https://hal.science/hal-01897341}

Submitted on 17 Oct 2018

HAL is a multi-disciplinary open access archive for the deposit and dissemination of scientific research documents, whether they are published or not. The documents may come from teaching and research institutions in France or abroad, or from public or private research centers.
L'archive ouverte pluridisciplinaire HAL, est destinée au dépôt et à la diffusion de documents scientifiques de niveau recherche, publiés ou non, émanant des établissements d'enseignement et de recherche français ou étrangers, des laboratoires publics ou privés. 


\title{
Cluster $\mathcal{X}$-varieties at infinity
}

\author{
V.V. Fock, A. B. Goncharov
}

To Joseph Bernstein for his 70th birthday

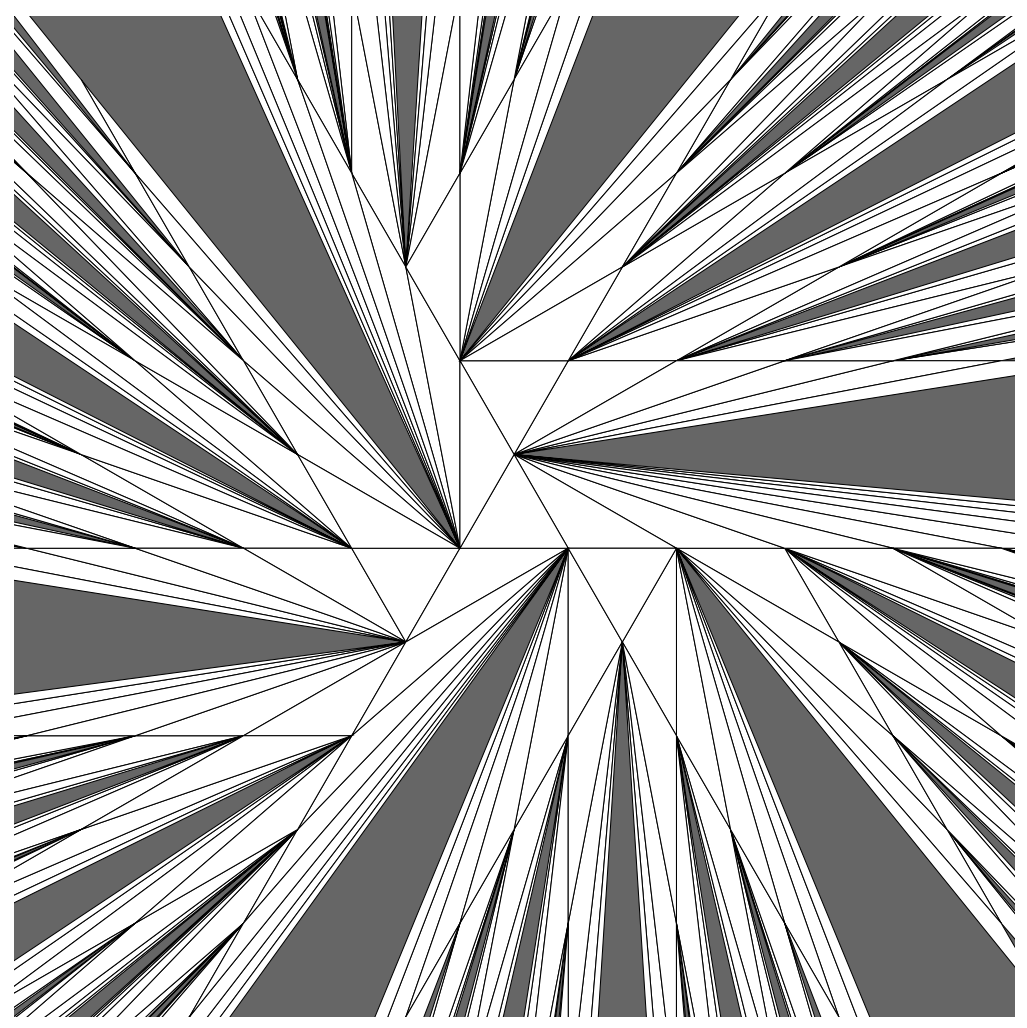

Figure 1: The tropical boundary hemisphere of the Teichmüller space of the punctured torus.

\section{Contents}

1 Introduction $\quad 2$

1.1 Geometric motivation: Thurston's boundary and completion of Teichmüller space

1.2 Algebraic perspective: positive spaces and cluster Poisson varieties. . . . . . . . .

1.3 Tropical compactifications for positive spaces. . . . . . . . . . . . . . 6

1.4 Special completions of cluster Poisson varieties. . . . . . . . . . . . 7

2 Tropical boundary and special completions of cluster Poisson varieties. 9

2.1 Tropical boundary of a positive space. . . . . . . . . . . . . 9 
2.2 Tropical boundary of the enhanced Teichmuller space of a punctured torus . . . . 10

2.3 Special completions of cluster varieties . . . . . . . . . . . . . . . 12

2.4 Convexity in the framework of positive spaces. . . . . . . . . . . . 17

\begin{abstract}
A positive space is a space with a positive atlas, i.e. a collection of rational coordinate systems with subtraction free transition functions. The set of positive real points of a positive space is well defined. We define a tropical compactification of the latter. We show that it generalizes Thurston's compactification of a Teichmüller space.

A cluster Poisson variety $1 \mathcal{X}$ is covered by a collection of coordinate tori $\left(\mathbb{C}^{*}\right)^{n}$, which form a positive atlas of a specific kind. We define a special completion $\widehat{\mathcal{X}}$ of $\mathcal{X}$. It has a stratification whose strata are cluster Poisson varieties. The coordinate tori of $\mathcal{X}$ extend to coordinate affine spaces $\mathbb{A}^{n}$ in $\widehat{\mathcal{X}}$.

We define completions of Teichmüller spaces for decorated surfaces $\mathbb{S}$ with marked points at the boundary. The set of positive points of the special completion of the cluster Poisson variety $\mathcal{X}_{P G L_{2}, \mathbb{S}}$ related to the Teichmüller theory on $\mathbb{S}[$ FG1 is a part of the completion of the Teichmüller space.
\end{abstract}

\title{
1 Introduction
}

\subsection{Geometric motivation: Thurston's boundary and completion of Teichmüller space}

Let $S$ be a closed oriented topological surface with $g>1$ handles. The Teichmüller space $\mathcal{T}_{S}$ of $S$ is isomorphic to $\mathbb{R}^{6 g-6}$. Thurston defined a compactification of the Teichmüller space by the space of projective measured laminations on $S$. The latter space is homeomorphic to a sphere $S^{6 g-7}$. The action of the modular group $\Gamma_{S}$ of $S$ extends to the Thurston compactification.

On the other hand, there is a completion $\mathcal{C}_{S}$ of the Teichmüller space, studied by L.Bers, H. Masur [M] and others. The action of the modular group $\Gamma_{S}$ on the Teichmüller space extends nicely to $\mathcal{C}_{S}$. The quotient $\mathcal{C}_{S} / \Gamma_{S}$ is identified with the Deligne-Mumford moduli space $\mathcal{M}_{g}$. The components of the boundary $\mathcal{C}_{S}-\mathcal{T}_{S}$ are parametrised by simple laminations on $S$, that is by the isotopy classes of collections of pairwise non-isotopic non-intersecting loops on $S$. These strata are of even real codimension - indeed, their quotients are complex varieties. Therefore the completion $\mathcal{C}_{S}$ is really different than the Thurston boundary.

One can extend the definition of the Teichmüller space and the Thurston compactification to the case when the closed surface $S$ is replaced by a decorated surface $\mathbb{S}$, given by a surface $S$ with a finite number of holes and a finite collection of marked points on the boundary, considered modulo isotopy. Below we reserve the notation $\mathbb{S}$ for decorated surfaces, using the notation $S$ for the surfaces without marked points on the boudnary. The enhanced Teichmüller space $\mathcal{T}_{\mathbb{S}}$ for a decorated syrface $\mathbb{S}$ parametrises complex structures on the underlying surface $S$ plus the following additional data: marked points, and choice of orientations for all boundary components without marked point:2. It is diffeomorphic to an open ball, and the Thurston compactification is homeomorphic to a closed ball. The Thurston boundary is identified with the projectivisation of the space of $\mathcal{X}$-laminations on $\mathbb{S}[$ FG3].

\footnotetext{
${ }^{1}$ originally called cluster $\mathcal{X}$-variety [FG2].

${ }^{2}$ When a hole is a puncture for the complex structure, its boundary is empty, so no orientation is involved.
} 
We define a natural completion $\mathcal{C}_{\mathbb{S}}$ of the enhanced Teichmüller space of $\mathbb{S}$, acted by the modular group of $\mathbb{S}$. In the special case when $\mathbb{S}$ reduces to a closed surface $S$ without holes, we recover the special completion $\mathcal{C}_{S}$ discussed above. For a decorated surface $\mathbb{S}$, the strata of the special completion $\mathcal{C}_{\mathbb{S}}$ are parametrised by simple $\mathcal{X}$-laminations on $\mathbb{S}$. However these strata are not necessarily of even codimension - they might be of codimension one, for example. Since we were not able to find a reference, we elaborate this below.

A boundary interval is a segment of the boundary connecting two neighboring marked points. A simple $\mathcal{X}$-lamination on $\mathbb{S}$ is a collection of pairwise non-intersecting loops and non-boundary, i.e. non-homotopic to a boundary interval, paths connecting unmarked boundary points, modulo isotopies. The boundary component corresponding to a single simple loop is a real codimension two stratum obtained by pinching the loop. The boundary component corresponding to a single non-boundary path $E$ connecting boundary points is a real codimension one stratum. It is identified with the enhanced Teichmüller space $\mathcal{T}_{\mathbb{S}_{E}}$ of the surface $\mathbb{S}_{E}$ obtained by cutting the original surface along $E$, see Fig 2. The marked points on $\mathbb{S}_{E}$ are the ones inherited from $\mathbb{S}$ plus the midpoints of the two components of the cutted segment $E$. There is a canonical fibration $\pi_{E}: \mathcal{T}_{\mathbb{S}} \longrightarrow \mathcal{T}_{\mathbb{S}_{E}}$ over the boundary component with one-dimensional fibers. More details can be found in Section 2.3.

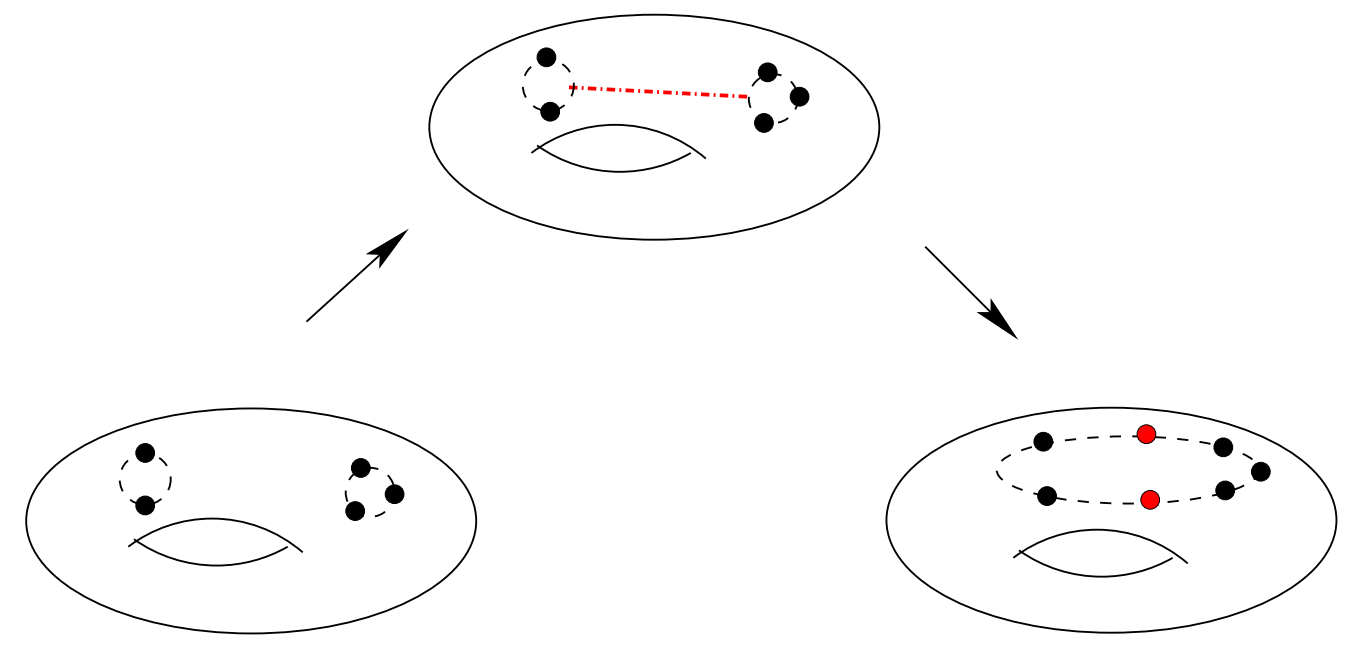

Figure 2: Starting with a torus with two holes and three marked points on the left, we cut it by a path $E$, shown on the top, getting a surface with one hole and five marked points.

Example. Let $S$ be a genus $g$ oriented hyperbolic surface with $n$ holes. The classical Teichmuller space $\mathcal{T}_{S}^{\text {un }}$ parametrises complex structures on $S$ such that each hole is a puncture for the complex structure. Its quotient under the action of modular group of $S$ is the moduli space $\mathcal{M}_{g, n}$. The quotient of the completion of the Teichmuller space $\mathcal{T}_{S}^{\text {un }}$ is the Deligne-Mumford moduli space $\overline{\mathcal{M}}_{g, n}$ : a regular projective scheme over $\mathbb{Z}$ compactifying the moduli space $\mathcal{M}_{g, n}$. The enhanced Teichmuller space $\mathcal{T}_{S}$ is bigger: $\operatorname{dim} \mathcal{T}_{S}=\operatorname{dim} \mathcal{T}_{S}^{\text {un }}+n$. It does not have a complex structure. Its completion has codimension one boundary components which do not intersect the completion of $\mathcal{T}_{S}^{\text {un }}$. For example, if $g=n=1$, the classical Teichmuller space $\mathcal{T}_{S}^{\text {un }}$ is the hyperbolic plane. Its completion is obtained by adding cusps at the boundary of the hyperbolic plane. The completion of the enhanced Teichmuller space $\mathcal{T}_{S}$ is more complicated. It is discussed in Section 2.2. 
The enhanced Teichmüller space of an arbitrary decorated surface $\mathbb{S}$ has an algebraicgeometric avatar: the moduli space $\mathcal{X}_{P G L_{2}, \mathbb{S}}$ of $P G L_{2}$-local systems on the underlying surface $S$ equipped with some additional structure near the boundary. The Teichmüller space is recovered as the space of its positive real points [FG1]. The moduli space $\mathcal{X}_{P G L_{2}, \mathbb{S}}$ has a natural cluster Poisson variety structure. So one may ask whether / how one can see the Thurston compactification and components of the completion of the Teichmüller space in terms of the cluster structure.

In this paper we address these questions from a quite general algebraic perspective. We show that the Thurston compactification is a special case of a very general construction which can be applied to an arbitrary positive space 3 Furthermore, the components of the completion of the Teichmüller space corresponding to simple $\mathcal{X}$-laminations without loops can be defined in the context of arbitrary cluster Poisson varieties.

\subsection{Algebraic perspective: positive spaces and cluster Poisson varieties.}

Many interesting varieties / moduli spaces admit natural positive atlases. A positive atlas is a collection of rational coordinate systems on a space such that the transition functions between any two of the coordinate systems are subtraction free rational functions 4 . The story originates in the classical theory of totally positive matrices. Further examples are discussed in [L, [FZ], FG1.

Example. The moduli space $\mathcal{M}_{0, n+3}$ parametrizes $(n+3)$-tuples of distinct points $\left(x_{1}, \ldots, x_{n+3}\right)$ on $\mathbb{P}^{1}$ modulo the action of $P G L_{2}$. A cyclic order of the points gives rise to a positive atlas on $\mathcal{M}_{0, n+3}$ as follows. Let $P_{n+3}$ be a convex polygon. We assign the points $x_{i}$ to its vertices, so that the order of points $x_{i}$ is compatible with the clockwise cyclic order of the vertices. The coordinate systems are parametrized by the triangulations of the polygon. Given a triangulation $T$, the coordinates $X_{E}^{T}$ are assigned to the diagonals $E$ of $T$. Namely, consider the unique rectangle formed by the sides of the polygon and diagonals of the triangulation, with the diagonal given by $E$. Its vertices provide a cyclic configuration of four points on $\mathbb{P}^{1}$. We order them starting from a vertex of $E$, getting a configuration of four points $\left(y_{1}, y_{2}, y_{3}, y_{4}\right)$ on $\mathbb{P}^{1}$, and set

$$
X_{E}^{T}:=r^{+}\left(y_{1}, y_{2}, y_{3}, y_{4}\right):=\frac{\left(y_{1}-y_{2}\right)\left(y_{3}-y_{4}\right)}{\left(y_{2}-y_{3}\right)\left(y_{1}-y_{4}\right)}, \quad r^{+}(\infty,-1,0, z)=z .
$$

There are two possible orderings, which differ by a cyclic shift by two. Since the cyclic shift by one changes the cross-ratio to its inverse, the rational function $X_{E}^{T}$ is well defined. The obtained coordinate systems form a positive atlas. Different dihedral orders of the points lead to different positive atlases. For example, there are 12 different positive atlases on $\mathcal{M}_{0,5}$. Each consists of five coordinate systems.

A positive space is a space $X$ together with a positive atlas. It is useful to separate the space from the atlas. Namely, the atlas itself determines a new scheme $\mathcal{X}$ over $\mathbb{Z}$ (perhaps non-separated). Let us describe the set of its complex points. Take a copy of the complex

\footnotetext{
${ }^{3}$ The analog of the Thurston boundary of a positive space $\mathcal{X}$ was defined in Section 4 of FG1. However the way it is attached to the positive real part of $\mathcal{X}$ - the analog of the Teichmüller space - was not discussed properly.

${ }^{4}$ These are the functions obtained from the coordinates by the operations of addition, multiplication and division, e.g. $\left(x+y^{2}\right) /\left(2 x^{3}+x y\right)$. Precise definitions are given in Section 1.1 of [FG2]. In particular, the space is rational.
} 
torus $\mathrm{T}_{\alpha}=\left(\mathbb{C}^{*}\right)^{n}$ assigned to each coordinate system $\alpha$ of the atlas. The canonical coordinates $X_{1}, \ldots, X_{n}$ on this torus are the coordinates of the coordinate system $\alpha$. Gluing these tori using the transition functions between the coordinate systems of the atlas we get a scheme $\mathcal{X}$ "materialising" the atlas. So a positive atlas on $X$ is nothing else but a birational isomorphism 5

$$
\psi: \mathcal{X} \longrightarrow X
$$

It turns out that positive atlases appearing in many interesting cases are of very specific nature: the whole atlas is determined by a single coordinate system equipped with certain additional structure. The original examples were provided by cluster algebras [FZI]. In this paper we consider such positive atlases of different type. The schemes materialising these atlases are called cluster Poisson varieties. They come with a natural Poisson structure.

Cluster Poisson varieties. Let us recall their definition, see Sections 1.2.1-1.2.4 of [FG2].

Definition 1.1. A quiver $\mathbf{s}$ is a datum $\left(\Lambda,\left\{e_{i}\right\},(*, *),\left\{d_{i}\right\}\right)$, where

- $\Lambda$ is a lattice, i.e. a free abelian group, $\left\{e_{i}\right\}$ is a basis of $\Lambda$, and $d_{i} \in \mathbb{Z}_{>0}$ are multipliers;

- $(*, *)$ a $\mathbb{Z}$-valued bilinear form on $\Lambda$ such that $\left\langle e_{i}, e_{j}\right\rangle:=\left(e_{i}, e_{j}\right) d_{j}^{-1}$ is skew-symmetric.

A quiver $\mathbf{s}$ can equivalently be described by a skewsymmetrisable matrix $\varepsilon_{i j}:=\left(e_{i}, e_{j}\right), i \in \mathrm{I}$.

Definition 1.2. A mutation of a quiver $\mathbf{s}$ in the direction of a basis vector $e_{k}$ is a new quiver $\widetilde{\mathbf{s}}$. The lattice and the form of the quiver $\widetilde{\mathbf{s}}$ are the same as of $\mathbf{s}$. The basis $\left\{\widetilde{e}_{i}\right\}$ of $\widetilde{\mathbf{s}}$ is defined by

$$
\widetilde{e}_{i}:= \begin{cases}e_{i}+\left(e_{i}, e_{k}\right)_{+} e_{k} & \text { if } i \neq k \\ -e_{k} & \text { if } i=k .\end{cases}
$$

The composition of mutations related to $e_{k}$ and $\widetilde{e}_{k}$ is an isomorphism of quivers. A composition of quiver mutations and quiver isomorphisms is called a quiver cluster transformation.

We assign to a quiver $\mathbf{s}$ a split torus $\mathrm{T}_{\mathbf{s}}:=\left(\mathbb{C}^{*}\right)^{\mathrm{I}}$, called a quiver torus, equipped with canonical coordinates $\left\{X_{i}\right\}, i \in \mathrm{I}$. A mutation in the direction of a basis vector $e_{k}$ gives rise to a birational isomorphism $\mu_{e_{k}}: \mathrm{T}_{\mathbf{s}} \longrightarrow \mathrm{T}_{\widetilde{\mathbf{s}}}$, acting on the set of coordinates $\left\{\widetilde{X}_{i}\right\}$ of $\mathrm{T}_{\widetilde{\mathbf{s}}}$ by

$$
\mu_{e_{k}}^{*}: \tilde{X}_{i} \longmapsto\left\{\begin{array}{lll}
X_{k}^{-1} & \text { if } & i=k, \\
X_{i}\left(1+X_{k}^{-\operatorname{sgn}\left(\varepsilon_{i k}\right)}\right)^{-\varepsilon_{i k}} & \text { if } & i \neq k .
\end{array}\right.
$$

An isomorphism of quivers gives rise to an isomorphism of the corresponding quiver tori. Thus a quiver cluster transformation gives rise to a birational isomorphism of the quiver tori.

Given a quiver s, we define the associated cluster Poisson variety as follows. Consider all quivers obtained from $\mathbf{s}$ by quiver cluster transformations. We assign to each of these quivers the corresponding quiver torus, and glue them using the corresponding birational isomorphisms.

One can deduce from the Laurent Phenomenon Theorem [FZL] that if the form $(*, *)$ is non-degenerate, the regular functions on a cluster Poisson variety separate its points.

\section{Examples.}

\footnotetext{
${ }^{5}$ We require it to satisfy an extra condition: the points of the torus $\mathrm{T}_{\alpha}$ where the map $\psi$ is not defined are contained in a divisor given by a positive equation. So in particular the restriction of $\psi$ to the positive part $\mathbb{R}_{>0}^{n}$ of the torus $\mathrm{T}_{\alpha}$ is well defined. Therefore we get a well defined subset $X\left(\mathbb{R}_{>0}\right)$ of positive real points, sitting inside of the set $X(\mathbb{R})$ positive points of $X$. Furthermore, $X\left(\mathbb{R}_{>0}\right)$ is identified, in many different ways, with $\mathbb{R}_{>0}^{n}$.
} 
1. The cluster Poisson variety $\mathcal{X}_{\Delta}$ provided by a classical Dynkin diagrams $\Delta$, i.e. $\Delta=A_{n}$. Its matrix $\varepsilon_{i j}$ is obtained by taking the Cartan matrix of the Dynkin diagrams $\Delta$, and making it skewsymmetrizable by killing the 2's on the diagonal and changing signs under the diagonal.

2. The positive atlas on $\mathcal{M}_{0, n+3}$ assigned to a cyclic order of the points is a cluster Poisson variety atlas. Namely, let us define a quiver assigned to a triangulation $T$. The lattice $\Lambda$ is the free abelian group generated by the diagonals of the triangulation, with a basis given by the diagonals. The bilinear form is given by the adjacency matrix. Namely, two diagonals $E$ and $F$ of the triangulation are called adjacent if they share a vertex, and there are no diagonals of the triangulation between them. We set $\varepsilon_{E F}=0$ if $E$ and $F$ are not adjacent. If they are, $\varepsilon_{E F}=1$ if $E$ is before $F$ according to the clockwise orientation of the diagonals at the vertex $v$ shared by $E$ and $F$, and $\varepsilon_{E F}=-1$ otherwise. Mutations correspond to flips of the diagonals. One shows that the adjacency matrix changes under the flips according to formula (3), and that the coordinates $X_{E}^{T}$ mutate according to formulae (4). A zig-zag triangulation of $P_{n+3}$ provides a quiver of type $A_{n}$.

3. The moduli space $\mathcal{X}_{G, S}$ parametrising $G$-local systems on an oriented surface $S$ with punctures, equipped with a reduction of the structure group to a Borel subgroup near every puncture. Here $G$ is a split reductive algebraic group over $\mathbb{Q}$ with connected center, e.g. $P G L_{n}$ [FG1]. The moduli space assigned to a decorated surface $\mathbb{S}$ is discussed in Section 2.3 .

So for each of these moduli spaces there is a cluster variety $\mathcal{X}$ and a birational isomorphism (2) describing the cluster atlas. However the map $\psi$ is almost never an isomorphism.

In Section 2 we investigate the following two problems:

1. How one can compactify the set of real positive points of an arbitrary positive space?

2. How one can complete cluster Poisson varieties by adding some "divisors at infinity"?

It turns out that these two problems are related.

\subsection{Tropical compactifications for positive spaces.}

Let us recall a few more notions related to positive spaces.

Let $\mathbb{F}$ be a semifield, i.e. a set with operations of addition, multiplication and division satisfying the usual axioms, e.g. $\mathbb{F}=\mathbb{R}_{>0}$. A more exotic example is the tropical semifield $\mathbb{R}^{t}$. This is the set $\mathbb{R}$ with the multiplication $\otimes$ and addition $\oplus$ given by $a \otimes b:=a+b, \quad a \oplus b:=$ $\max (a, b)$. Similarly there are tropical semifields $\mathbb{Z}^{t}$ and $\mathbb{Q}^{t}$.

A positive rational function provides a map of a semifield $\mathbb{F}$ to itself. Therefore a positive space $X$ determines a set $X(\mathbb{F})$ of its $\mathbb{F}$-valued points. As a set, it is isomorphic, in many different ways, to the set $\mathbb{F}^{\operatorname{dim} X}$. Namely, we assign to each coordinate system of the atlas the set $\mathbb{F}^{\operatorname{dim} X}$, and glue them using the coordinate transformations. The group of automorphisms of the positive space $X$ acts on the set $X(\mathbb{F})$. If the space is a cluster Poisson variety $\mathcal{X}$, there is a distinguished subgroup of the latter, called the cluster modular group $\Gamma_{\mathcal{X}}$ of $\mathcal{X}$.

A positive space $\mathcal{X}$ provides us with two spaces: the space of real positive points $\mathcal{X}\left(\mathbb{R}_{>0}\right)$ and the space of real tropical points $\mathcal{X}\left(\mathbb{R}^{t}\right)$. 
Example [FG1]. The set $\mathcal{X}_{G, S}\left(\mathbb{R}_{>0}\right)$ of positive points of the moduli space $\mathcal{X}_{G, S}$ is the higher Teichmüller space assigned to the pair $(G, S)$. For $G=P G L_{2}$ we recover the enhanced Teichmüller space of $S$. The set $\mathcal{X}_{P G L_{2}, S}\left(\mathbb{R}^{t}\right)$ is the space of measured $\mathcal{X}$-laminations on $S$.

In Section 2 we relate the spaces $\mathcal{X}\left(\mathbb{R}_{>0}\right)$ and $\mathcal{X}\left(\mathbb{R}^{t}\right)$ as follows. We define the tropical compactification of the space $\mathcal{X}\left(\mathbb{R}_{>0}\right)$. Its boundary is called the tropical boundary of $\mathcal{X}\left(\mathbb{R}_{>0}\right)$. We show that the tropical boundary is canonically identified with the spherical tropical space $\mathcal{S} \mathcal{X}\left(\mathbb{R}^{t}\right)$ - the quotient of $\mathcal{X}\left(\mathbb{R}^{t}\right)$ by the natural action of the group $\mathbb{R}_{>0}^{*}$.

Example. We show that the tropical boundary of the positive space $\mathcal{X}_{P G L_{2}, S}$ is the Thurston boundary of the classical Teichmüller space. Since the spherical tropical space of this positive space is the space of projective measured laminations on $S$, our theorem in this case reduces to Thurston's theorem.

The spherical tropical space $\mathcal{S} \mathcal{X}\left(\mathbb{R}^{t}\right)$ is a sphere with a piecewise linear structure preserved by the action of the automorphism group $\Gamma_{\mathcal{X}}$. It is a rather complicated object: Fig 1 shows a hemisphere of the two dimensional spherical tropical space for $\mathcal{X}_{P G L_{2}, S}$, where $S$ is a torus with a hole. The automorphism group $\Gamma_{\mathcal{X}}=P S L_{2}(\mathbb{Z})$ acts by piecewise linear transformations of the hemisphere. So one can decompose it into the maximal domains where the action is linear, as shown on Fig 1. We discuss this in detail in Section 2.2 .

In Section 2.4 we introduce a convex structure on the spherical tropical space $\mathcal{S} \mathcal{X}\left(\mathbb{R}^{t}\right)$.

\subsection{Special completions of cluster Poisson varieties.}

We show that the rational points the spherical tropical space parametrize a supply of divisors which can appear as divisors at infinity. To specify a particular completion we need to choose a "fan" in the tropical space, which is reminiscent of the definition of toric varieties.

An arbitrary cluster Poisson variety gives rise to a canonical "fan" in the tropical space $\mathcal{X}\left(\mathbb{R}^{t}\right)$. Namely, we assign to a coordinate system a cone in $\mathcal{X}\left(\mathbb{R}^{t}\right)$ consisting of all real tropical points which have non-negative coordinates in this coordinate system. We call it a positive cone.

Conjecture 1.3. The interiers of the positive cones assigned to different cluster $\mathcal{X}$-coordinate systems are disjoint.

The union of these cones is the positive part of the tropical space.

The positive cone gives rise to a special completion $\widehat{\mathcal{X}}$ of the cluster Poisson variety. The strata of $\widehat{\mathcal{X}}$ are cluster Poisson varieties. The codimension one strata are described as follows. Each cluster coordinate $X_{k}$ gives rise to a codimension one cluster variety on the boundary $\widehat{\mathcal{X}}-\mathcal{X}$. Cluster coordinates $X_{k}$ and $X_{k}^{\prime}$ produce the same boundary component if and only if there is a cluster transformation $\mu_{i_{1}} \circ \ldots \ldots \circ \mu_{i_{n}}$ with $i_{1}, \ldots, i_{n} \in \mathrm{I}-\{k\}$, which transforms $X_{k}$ to $X_{k}^{\prime}$. This boundary component is given by the equation $X_{k}=0$. The key observation is that, as it is clear from (44), a cluster Poisson transformation $\mu_{j}$ at $j \in \mathrm{I}-\{k\}$ transforms the equation $X_{k}=0$ to the one $X_{k}^{\prime}=0$. This boundary cluster Poisson variety is determined by a quiver described by the restriction of the matrix $\varepsilon_{i j}$ to $i, j \in \mathrm{I}-\{k\}$. The Poisson structure on

$\mathcal{X}$ extends to the special completion $\widehat{\mathcal{X}}$, so that the divisor at infinity is precisely the subscheme of zeros of the Poisson bivector field.

One should not mix up tropical compactifications and cluster completions. The latter are algebraic geometric objects, while the former are piecewise linear spheres. They are related 
however: the rational points of the tropical boundary parametrise a supply of divisors at infinity which can appear in different completions.

One can take points of the special completion $\widehat{\mathcal{X}}$ with values in any semifield.

Examples. 1. For the cluster variety $\mathcal{X}_{A_{n}}$ the boundary of the special completion is a divisor combinatorially equivalent to the boundary of the $n$-dimensional Stasheff polytope, which we will discuss in detal below; the tropical boundary $\mathcal{S} \mathcal{X}_{A_{n}}\left(\mathbb{R}^{t}\right)$ is a simplicial sphere $S^{n-1}$ dual to the boundary of the $n$-dimensional Stasheff polytope; and $\widehat{\mathcal{X}}_{A_{n}}\left(\mathbb{R}_{>0}\right)$ is the Stasheff polytope. So there are two completely different ways to compactify the moduli space $\mathcal{X}_{A_{n}}\left(\mathbb{R}_{>0}\right)$ of positive configurations of points on $\mathbb{R P}^{1}$, although topologically they both are spheres. We continue discussion of this example in Section 2.3.

2. The set of positive points of the special completion of the moduli space $\mathcal{X}_{P G L_{2}, S}$ is a stratified space. Its codimension $k$ strata are the Teichmüller spaces for the decorated surfaces $\mathbb{S}$ obtained by cutting the original surface $S$ along $k$ non-intersecting ideal edges.

3. Toric varieties. A quiver with the trivial bilinear form is just a lattice with a basis. The special completion in this case is a product of $\mathbb{P}^{1}$ 's. Other completions are toric varieties.

In general the special completion $\widehat{\mathcal{X}}\left(\mathbb{R}_{>0}\right)$ of the space $\mathcal{X}\left(\mathbb{R}_{>0}\right)$ and the tropical compactification of $\mathcal{X}\left(\mathbb{R}_{>0}\right)$ are different: the latter is a sphere, while the former may not be compact.

The positive part of the tropical boundary in general fails to be a simplicial complex since some faces may be shared by infinitely many special simplices. The complement

(the tropical boundary) - (the positive part of the tropical boundary)

is an interesting object. It leads, at least in some cases, to generalizations of cluster coordinates.

For example, for the boundary of the Teichmuller space on one punctured torus, depicted on Fig 1 , the complement is a union of "black rays" parametrised by the $\mathrm{P}^{1}(\mathbb{Q})$. They are the limits of the "thin black cones", which are artefacts of the resolution of the picture. The "black rays" are in fact parametrised naturally by the cusps on the boundary of the hyperbolic plane.

The following conjecture is a more precise version of Conjecture 1.3 ,

Conjecture 1.4. The cluster complex (Definition 2.14 in [FG2]) is naturally identified with a connected component of the positive part of the tropical boundary.

Moduli spaces of the Stokes data and degenerations. A $G$-bundle with meromorphic connection on a Riemann surface $\Sigma$, with possibly irregular singularities, is determined by a generalized monodromy data, which consists of the monodromy and the Stokes data at irregular singularities. When $G=P G L_{2}$, the moduli space $\mathcal{M S}(\Sigma)$ of the generalized monodromy data has a cluster Poisson variety structure, and is isomorphic to $\mathcal{X}_{P G L_{2}, \mathbb{S}}$, where $S$ is the topological surface underlying the $\Sigma$, and the isotopy class of marked boundary points on the boundary of $S$ is determined by the irregularity type of the connection. The strata of the special completion of generalized monodromy moduli space $\mathcal{M S}(\Sigma)$ are obtained by colliding the singularities of meromorphic connections on $\Sigma$. A similar result is valid for any reductive group $G$, and will be discussed in detail elsewhere. 
Acknowledgments. A.G. was supported by the NSF grants DMS-0653721, DMS-1059129 and DMS-1301776. The first (repectively the final) draft of this paper was written when A.G. enjoyed the hospitality of IHES (Bures sur Yvette) in 2009 (repectively in 2015). He is grateful to IHES for the support.

We are very grateful to the referee for correction of some of our errors, and useful comments.

\section{Tropical boundary and special completions of cluster Poisson varieties.}

\subsection{Tropical boundary of a positive space.}

Let $\mathcal{X}$ be a positive space. A positive rational function on $\mathcal{X}$ is a rational function on $\mathcal{X}$ which in some, and hence any positive coordinate system can be presented as a ratio of two Laurent polynomials with positive integral coefficients. Positive rational functions form a semifield denoted by $\mathbb{Q}_{+}(\mathcal{X})$. A positive rational function $F$ takes positive values on any positive point $x \in \mathcal{X}\left(\mathbb{R}_{>0}\right)$. So $\log F(x)$ is defined. Choose a (countable) basis $\mathbf{B}$ in the semigroup $\mathbb{Q}_{+}(\mathcal{X})$. The logarithms of values of functions $F \in \mathbf{B}$ on positive points provide a map

$$
\mathcal{X}\left(\mathbb{R}_{>0}\right) \longrightarrow \mathbb{R}^{\mathbf{B}}, \quad x \longmapsto\{\log F(x)\}_{F \in \mathbf{B}} .
$$

The projectivisation of this map is a map, obviously injective,

$$
\tau: \mathcal{X}\left(\mathbb{R}_{>0}\right) \hookrightarrow \mathbb{P}\left(\mathbb{R}^{\mathbf{B}}\right) .
$$

Definition 2.1. Let $\mathcal{X}$ be a positive space. The tropical compactification $\overline{\mathcal{X}^{+}}$of the space $\mathcal{X}\left(\mathbb{R}_{>0}\right)$ is the closure of the subset

$$
\tau\left(\mathcal{X}\left(\mathbb{R}_{>0}\right)\right) \subset \mathbb{P}\left(\mathbb{R}^{\mathbf{E}}\right) .
$$

The tropical boundary of the space $\mathcal{X}\left(\mathbb{R}_{>0}\right)$ is the complement $\partial \overline{\mathcal{X}^{+}}:=\overline{\mathcal{X}^{+}}-\mathcal{X}^{+}$.

Spherical tropical space. The group $\mathbb{R}_{>0}^{*}$ acts on the set $\mathcal{X}\left(\mathbb{R}^{t}\right)$ of real tropical points of $\mathcal{X}$. A number $\lambda \in \mathbb{R}_{>0}^{*}$ acts by multiplying all coordinates at some (and hence any) positive coordinate system by $\lambda$. The quotient of $\mathcal{X}\left(\mathbb{R}^{t}\right)$ by this action is the spherical tropical space $\mathcal{S} \mathcal{X}\left(\mathbb{R}^{t}\right)$. Obviously,

$$
\mathcal{S} \mathcal{X}\left(\mathbb{R}^{t}\right) \text { is piecewise linear isomorphic to } S^{n-1}, \quad n=\operatorname{dim}(\mathcal{X}) .
$$

Proposition 2.2. There is a natural isomorphism

$$
\eta: \mathcal{S X}\left(\mathbb{R}^{t}\right) \stackrel{\sim}{\longrightarrow} \partial \overline{\mathcal{X}^{+}} .
$$

Proof. Let us define the map $\eta$. Observe that

$$
\lim _{C \rightarrow \infty} \frac{\log \left(e^{C x_{1}}+\ldots+e^{C x_{n}}\right)}{C}=\max \left\{x_{1}, \ldots, x_{n}\right\} .
$$

Thus the evaluation of a rational positive function $F$ on a tropical point $x \in \mathcal{X}\left(\mathbb{R}^{t}\right)$ can be interpreted as follows. Take a positive coordinate system. Let $\left(x_{1}, \ldots, x_{n}\right)$ be the coordinates of $x$. Then

$$
F^{t}(x)=\lim _{C \rightarrow \infty} \frac{\log F\left(e^{C x_{1}}, \ldots, e^{C x_{n}}\right)}{C} .
$$


It remains to notice that in the projective space

$$
\left(\frac{\log F_{1}\left(e^{C x_{1}}, \ldots\right)}{C}: \frac{\log F_{2}\left(e^{C x_{1}}, \ldots\right)}{C}: \ldots\right)=\left(\log F_{1}\left(e^{C x_{1}}, \ldots\right): \log F_{2}\left(e^{C x_{1}}, \ldots\right): \ldots\right) .
$$

So we get an injective map $\eta$. Let us show that it is surjective. Choose a positive coordinate system. Let $\left(x_{1}(\varepsilon), \ldots, x_{n}(\varepsilon)\right)$ be the coordinates of a family of points of $\mathcal{X}\left(\mathbb{R}_{>0}\right)$ which has a limit under the map $\tau$ to the projective space. Consider its image in the subspace of the projective space $\mathbb{P}\left(\mathbb{R}^{\mathbf{E}}\right)$ corresponding to the coordinate functions. Let $\left(x_{1}: \ldots: x_{n}\right)$ be the homogeneous coordinate of the limiting point, which we assume for a moment being defined, i.e. different then $(0:, \ldots,: 0)$. Then the limiting point is of form $\eta\left(x_{1}: \ldots: x_{n}\right)$. The proposition is proved.

Example. Let $S$ be a surface with punctures. According to [FG1, the space of projective measured laminations coincides with spherical tropical space for $\mathcal{X}_{P G L_{2}, S}$.

Let $\mathcal{L}$ be the set of isotopy classes of simple loops on $S$. The Thurston compactification of the Teichmüller space is defined as the closure of the image of the map

$$
\mathcal{T}_{S} \longrightarrow \mathbb{P}\left(\mathbb{R}^{\mathcal{L}}\right)
$$

of the Teichmuller space into the infinite dimensional real projective space, defined as follows. A point $x \in \mathcal{T}_{S}$ is assigned a point in the projective space whose homogeneous coordinates are the lengths of the loops in the metric on $S$ given by the point $x \in \mathcal{T}_{S}$.

Proposition 2.3. The Thurston boundary of the Teichmüller space $\mathcal{T}_{S}$ coincides with the tropical boundary of the cluster Poisson variety $\mathcal{X}_{P G L_{2}, S}$.

Proof. The proposition is an easy consequence of the following two facts, proved in [FG1]. First, a loop $\alpha$ on $S$ gives rise to a positive regular function on the space $\mathcal{X}_{P G L_{2}, S}$ provided by the local system monodromy along $\alpha$. Second, let $\lambda_{1}, \lambda_{2}$ be the eigenvalues of the monodromy around $\alpha$ for the $P G L_{2}(\mathbb{R})$-local system assigned to a point $x \in \mathcal{T}_{S}$. They are defined up to a common factor. Then the length of the geodesic representing the isotopy class of $\alpha$ is $\frac{1}{2} \log \left|\lambda_{1} / \lambda_{2}\right|$.

\subsection{Tropical boundary of the enhanced Teichmuller space of a punctured torus}

The (enhanced) Teichmuller space for the punctured torus has dimension 3. It is the space of positive points for the cluster Poisson variety structure described by the matrix

$$
\varepsilon_{i j}=\left(\begin{array}{ccc}
0 & 2 & -2 \\
-2 & 0 & 2 \\
2 & -2 & 0
\end{array}\right)
$$

So its tropical boundary is a sphere $S^{2}$ equipped with a piecewise linear action of the mapping class group $P S L_{2}(\mathbb{Z})$. Below we describe this action, as well as the domains where it is linear.

A coordinate description. The group $P S L_{2}(\mathbb{Z})$ is generated by the standard generators $S$ and $T$, such that $S^{2}=(S T)^{3}=e$. The group $P S L_{2}(\mathbb{Z})$ acts by piecewise linear transformations of $\mathbb{Z}^{3}$ : The generator $S T$ acts by $(x, y, z) \longmapsto(y, z, x)$, and the generator $T$ acts by

$$
x \longmapsto x-2 \max (0,-z), \quad y \longmapsto y+2 \max (0, z), \quad z \longmapsto-z .
$$


The action preserves $x+y+z$, and hence the half spaces $x+y+z>0$ and $x+y+z<0$. To visualize it, we describe its restriction to the plane $x+y+z=1$. There is a triangle

$$
T=\{x, y, z \mid x, y, z \geq 0, x+y+z=1\} .
$$

The $P S L_{2}(\mathbb{Z})$-orbits of the triangle $T$ fill a dense part of the plane. Every side of the obtained triangles is shared by exactly two triangles. The complement is a countable collection of rays. Each ray has a unique vertex. Every vertex of the triangles is a vertex of a unique ray. Every vertex is shared by infinitely many triangles and just one ray.

A description via projective measured laminations. We consider on the punctured torus $S^{\prime}$ simple paths $\alpha$ without selfintersection of two types: ideal arcs, which start and end at the puncture, and loops.

A rational lamination on $S$ is the following data:

- a collection of mutually non-intersecting and non-isotopic ideal arcs and simple loops with positive rational weights on the surface $S^{\prime}$, up to isotopy.

- If a lamination contains an ideal arc, an orientation of a small disc containing the puncture.

Real laminations are defined as a completion of the space of rational laminations.

The space of projective measured laminations is identified with the sphere $S^{2}$ as follows.

There is a decomposition of the sphere $S^{2}$ into a union of a circle and two hemispheres:

$$
S^{2}=S^{1} \cup S^{+} \cup S^{-}
$$

The equatorial circle $S^{1}$ parametrises projective measured laminations which are limits of the rational laminations supported on a single loop, as we will see shortly.

The support of any other projective measured lamination contains an ideal arc. The sign of the hemisphere $S^{ \pm}$where the point parametrising such a projective measured lamination is located is determined by the orientation of a disc containing the puncture: it is + if it is induced by the orientation of $S^{\prime}$.

The triangles parametrise projective laminations

$$
a_{1} \alpha_{1}+a_{2} \alpha_{2}+a_{3} \alpha_{3}, \quad a_{1}, a_{2}, a_{3} \geq 0
$$

where the ideal arcs $\alpha_{1}, \alpha_{2}, \alpha_{3}$ form an ideal triangulation of the punctured torus $S^{\prime}$, and the triple $\left(a_{1}, a_{2}, a_{3}\right)$ is defined up to multiplication by a positive number. The edges of the triangles parametrise projective laminations of the form $a_{1} \alpha_{1}+a_{2} \alpha_{2}$, and the vertices correspond to the laminations given by the ideal $\operatorname{arcs} \alpha$ on $S^{\prime}$.

Rays parametrise projective laminations

$$
a \alpha+b \beta, \quad a, b \geq 0,
$$

where $\alpha$ is an ideal arc, $\beta$ is a loop, and the pair $(a, b)$ is defined up to multiplication by a positive number. The projective lamination $\beta$ corresponds to the intersection of the ray with the equatorial circle. The projective lamination $\alpha$ is the vertex of the ray shared by the triangles.

The equatorial circle $S^{1}$ parametrises projective measured laminations on the torus compactifying $S^{\prime}$. Presenting a compact torus as $\mathbb{R}^{2} / \mathbb{Z}^{2}$, the equatorial circle $S^{1}$ is identified with 
$\mathbb{R}^{2} / \mathbb{R}_{>0}^{*}$. A projective lamination is obtained by projecting a one dimensional subspace in $\mathbb{R}^{2}$ onto the torus. Integral laminations correspond to the lines with rational slopes, i.e. to the rational points $p / q$ on $S^{1}$. The action of the modular group $P S L_{2}(\mathbb{Z})$ on the projective measured laminations is the action by the fractional linear transformations of the equatorial circle $S^{1}$.

Let us compare the $S^{+} \cup S^{1}$ part of the tropical boundary on Fig [1 with the classical modular triangulation of the hyperbolic plane. There is a natural map from the former to the latter. It shrinks the rays onto the cusps, and is an isomorphism on the triangles.

\subsection{Special completions of cluster varieties}

Rational tropical points and strict valuations. A valuation on a ring $\mathbb{L}$ is a map $v$ : $\mathbb{L} \longrightarrow \mathbb{Z}$ such that

$$
v\left(L_{1} L_{2}\right)=v\left(L_{1}\right)+v\left(L_{2}\right), \quad v\left(L_{1}+L_{2}\right) \leq \min \left(v\left(L_{1}\right), v\left(L_{2}\right)\right) .
$$

A strict valuation on a semiring $\mathbb{L}_{+}$is a map $v: \mathbb{L}_{+} \longrightarrow \mathbb{Z}$ such that

$$
v\left(L_{1} L_{2}\right)=v\left(L_{1}\right)+v\left(L_{2}\right), \quad v\left(L_{1}+L_{2}\right)=\min \left(v\left(L_{1}\right), v\left(L_{2}\right)\right) .
$$

A valuation $v$ gives rise to the valuation semiring

$$
\mathcal{O}_{v}^{+} \subset \mathbb{L}_{+}, \quad \mathcal{O}_{v}=\left\{F \in \mathbb{L}_{+} \mid v(F) \geq 0\right\}
$$

and the valuation ideal

$$
\mathcal{M}_{v}^{+} \subset \mathcal{O}_{v}^{+}, \quad \mathcal{M}_{v}=\left\{F \in \mathbb{L}_{+} \mid v(F)>0\right\} .
$$

So there is the quotient semiring

$$
\mathcal{R}_{v}^{+}:=\mathcal{O}_{v}^{+} / \mathcal{M}_{v}^{+} .
$$

The following lemma is an immediate consequence of the definitions.

Lemma 2.4. Let $\mathcal{X}$ be a positive space. A point $x \in \mathcal{X}\left(\mathbb{Z}^{t}\right)$ provides a strict valuation $v_{x}$ of the semiring $\mathbb{L}_{+}(\mathcal{X})$ given by the negative of the value of the tropicalization of a function $F$ at $x$ :

$$
v_{x}(F):=-F^{t}(x) .
$$

Remark. Let $\mathcal{X}$ and $\mathcal{A}^{\vee}$ be a Langlands dual pair of cluster varieties - see Section 4 of [FG2]. Then the intersection pairing $\mathcal{I}_{\mathcal{A}}(l, m): \mathcal{A}^{\vee}\left(\mathbb{Z}^{t}\right) \times \mathcal{X}\left(\mathbb{Z}^{t}\right) \longrightarrow \mathbb{Z}$ whose existence was conjectured in Conjecture 4.3 in loc. cit. is nothing else but the value of the valuation $v_{m}$ provided by an integral tropical point $m \in \mathcal{X}\left(\mathbb{Z}^{t}\right)$ on the positive regular function $\mathbb{I}_{\mathcal{A}}(l)$ on the $\mathcal{X}$-space corresponding by Conjecture 4.1 in loc. cit. to the integral tropical point $l \in \mathcal{A}^{\vee}\left(\mathbb{Z}^{t}\right)$ :

$$
\mathcal{I}_{\mathcal{A}}(l, m)=v_{m}\left(\mathbb{I}_{\mathcal{A}}(l)\right) .
$$

There is, of course, a similar picture when we interchange $\mathcal{X}$ and $\mathcal{A}$ spaces. 
Divisors at infinity corresponding to the points of $\mathcal{S} \mathcal{X}\left(\mathbb{Q}^{t}\right)$. Let us assume that the ring $\mathbb{L}(\mathcal{X})$ is spanned by the semiring $\mathbb{L}_{+}(\mathcal{X})$, and the strict valuation $v_{x}$ on $\mathbb{L}_{+}(\mathcal{X})$ can be extended to a valuation on $\mathbb{L}(\mathcal{X})$, also denoted by $v_{x}$. So there are the valuation ring $\mathcal{O}_{x}$, the valuation ideal $\mathcal{M}_{x}$, and the quotient ring $\mathcal{R}_{x}$ for the valuation $v_{x}$. Then, given $x \in \mathcal{X}\left(\mathbb{Z}^{t}\right)$, there is a scheme

$$
\mathcal{D}_{x}:=\operatorname{Spec}\left(\mathcal{R}_{x}\right)
$$

We think about it as of a divisor at infinity for the space $\mathcal{X}$ corresponding to $x$. The integer $v_{x}(F)$ is the order of zero of the function $F$ at this divisor. The scheme $\mathcal{D}_{x}$ depends only on the image of $x$ in $\mathcal{S X}\left(\mathbb{Q}^{t}\right)$.

If $\mathcal{X}$ has a cluster variety, $\mathcal{D}_{x}$ does not always have a cluster variety structure. Here is an example which does not require any a priori assumptions, where this is the case.

Special completions of cluster varieties. Given a quiver $\mathbf{i}$, any subset of its basis vectors determines a quiver $\mathbf{j}$, called a subquiver of the quiver $\mathbf{i}$. Its lattice $\Lambda_{\mathbf{j}}$ is the sublattice of $\Lambda$ spanned by the specified basis vectors; the bilinear form on the lattice $\Lambda_{\mathbf{j}}$ is induced by the bilinear form on $\Lambda$.

Definition 2.5. Let $\mathcal{X}$ be a cluster Poisson variety. A special cone is a cone $C \subset \mathcal{X}\left(\mathbb{R}^{t}\right)$ such that there exists a quiver $\mathbf{i}$ and a subquiver $\mathbf{j} \subset \mathbf{i}$ so that $C$ is given by the following conditions on the tropical cluster coordinates $\left\{x_{i}\right\}$ for the quiver $\mathbf{i}$ :

$$
C=\left\{l \in \mathcal{X}\left(\mathbb{R}^{t}\right) \mid x_{i}(l) \geq 0, \quad x_{c}(l)=0 \text { if } c \in \mathbf{j}\right\} .
$$

So $C$ is a cone of codimension $|\mathbf{j}|$.

Remark. A special simplex is the projection of a special cone to the spherical tropical space. Special simplices may not form a locally finite subset of the spherical tropical space. An example is given in Section 2.2, where special triangles are the orbits of the triangle $T$.

Lemma 2.6. A special cone $C \subset \mathcal{X}\left(\mathbb{R}^{t}\right)$ determines a cluster Poisson variety $\mathcal{X}_{C}$ of codimension $\operatorname{dim} C$.

Proof. Mutating basis vectors of the subquiver $\mathbf{j}$ we do not change coordinates of a point $l \in C$. Indeed, the tropical coordinates $x_{i}$ change under the mutation in the direction $e_{j}$ as follows:

$$
x_{i}^{\prime}=x_{i} \pm \varepsilon_{i j} \max \left(0, \mp x_{j}\right), \quad i \neq j, \quad x_{j}^{\prime}=-x_{j} .
$$

So $x_{j}=0$ implies $x_{i}^{\prime}=x_{i}$ for all $i$. Therefore the equivalence class of the quiver $\mathbf{j}$ is well defined by the special cone $C$. The lemma is proved.

Proposition 2.7. Assume that regular functions on a cluster Poisson variety $\mathcal{X}$ separate the points. Then there is a unique scheme over $\mathbb{Z}$, perhaps non-separated, called the special completion $\widehat{\mathcal{X}}$ of $\mathcal{X}$, such that

1. $\widehat{\mathcal{X}}$ has a stratification whose strata are the affine closures of cluster varieties $\mathcal{X}_{C}$ parametrised by the special cones $C \subset \mathcal{X}\left(\mathbb{R}^{t}\right)$.

2. We have $\mathcal{X}_{C} \subset \widehat{\mathcal{X}}_{C^{\prime}}$ if and only if $C^{\prime} \subset C$. 
Proof. A quiver $\mathbf{i}$ defines a cluster quiver torus $\mathcal{X}_{\mathbf{i}}$ which is canonically identified with $\mathbb{G}_{m}^{\mathrm{I}}$ by the cluster coordinates. It has a canonical embedding into the affine space

$$
\mathcal{X}_{\mathbf{i}} \cong \mathbb{G}_{m}^{\mathrm{I}} \hookrightarrow \mathbb{A}^{\mathrm{I}}
$$

A subquiver $\mathbf{j} \subset \mathbf{i}$ determines a cluster torus $\mathcal{X}_{\mathbf{j}}$ which we glue to the torus $\mathcal{X}_{\mathbf{i}}$ as follows: the torus $\mathcal{X}_{\mathbf{j}}$ is realized as a subvariety of $\mathbb{A}^{\mathrm{I}}$ in (8) given by the conditions

$$
X_{j}=0 \text { if } j \in \mathbf{i}-\mathbf{j} \text {, and } X_{j} \neq 0 \text { otherwise. }
$$

The key point is that mutations in the directions of basis vectors of $\mathbf{j}$ do not change condition (91). Therefore it provides a boundary stratum identified with the cluster variety corresponding to the quiver $\mathbf{j}$. This procedure is transitive: given a triple of quivers $\mathbf{k} \subset \mathbf{j} \subset \mathbf{i}$, the stratum for the subquiver $\mathbf{k} \subset \mathbf{i}$ is the same as the stratum for the subquiver $\mathbf{k} \subset \mathbf{j}$ in the stratum for $\mathbf{j} \subset \mathbf{i}$. The proposition follows.

Remark. There is a version of Proposition 2.7 where we do not take affine closures in 1). It does not require that regular functions on $\mathcal{X}$ separate the points.

Taking the positive points of the boundary strata we get a completion $\widehat{\mathcal{X}}\left(\mathbb{R}_{>0}\right)$ of the space $\mathcal{X}\left(\mathbb{R}_{>0}\right)$. It consists of cells, each isomorphic to $\mathbb{R}^{k}$, parametrised by the special cones, where $k$ is the codimension of the cone.

For a cluster Poisson variety of finite type the completion $\widehat{\mathcal{X}}\left(\mathbb{R}_{>0}\right)$ is homeomorphic to a disc. Its boundary is a polyhedron dual to the generalized Stasheff polyhedron of the Dynkin diagram. Otherwise the special completion is not a manifold.

Here are some examples.

Special completion of the moduli space of framed $P G L_{2}$-local systems. Let $\mathbb{S}$ be a decorated surface. Let $\partial \mathbb{S}$ be its punctured boundary, i.e. the boundary minus the marked points. The moduli space $\mathcal{X}_{P G L_{2}, \mathbb{S}}$ parametrised pairs $(\mathcal{L}, \beta)$, called framed $P G L_{2}$-local systems, where $\mathcal{L}$ is a $P G L_{2}$-local system on $\mathbb{S}$, and $\beta$ is a flat section of the associated flat $\mathbb{P}^{1}$-bundle on $\partial \mathbb{S}$. It amounts to a collection of flat sections on connected components of the boundary. In the absence of marked points we get the moduli space described in the Example 3 at Section 1.2.

Choose a point inside of each connected component of $\partial \mathbb{S}$. An ideal triangulations of $\mathbb{S}$ is a triangulation of the surface with vertices at these points. The moduli space $\mathcal{X}_{P G L_{2}, \mathbb{S}}$ has a cluster Poisson variety structure with the quivers parametrised by ideal triangulations $T$ of $\mathbb{S}$. The cluster coordinates for a given triangulation $T$ are assigned to the non-boundary edges of $T$ [FG1], just like in the case of $\mathcal{M}_{0, n+3}$.

For example, when $\mathbb{S}$ is a disc with $n+3$ marked points at the boundary, the moduli space $\mathcal{X}_{P G L_{2}, \mathbb{S}}$ parametrises $(n+3)$-tuples of points $\left(x_{1}, \ldots, x_{n+3}\right)$ on $\mathbb{P}^{1}$ modulo the diagonal action of the group $P G L_{2}$. To decribe its cluster structure we start with a convex $(n+3)$-gon $P_{n+3}$. Its vertices are the marked points. We assign the points $\left(x_{1}, \ldots, x_{n+3}\right)$ to the sides of the polygon $P_{n+3}$ as on Fig 3. Now take the convex hull of the centers of the sides of the polygon $P_{n+3}$. We get a dual convex polygon, denoted by $* P_{n+3}$. The configuration of points $\left(x_{1}, \ldots, x_{n+3}\right)$ sits at its vertices. A triangulation of the dual polygon $* P_{n+3}$ is an ideal triangulation.

To get a boundary divisor, we cut the polygon $P_{n+3}$ along a line segment $E$ connecting midponits of two of its non-consequitive sides. So $E$ is a diagonal of the polygon $* P_{n+3}$. Then 


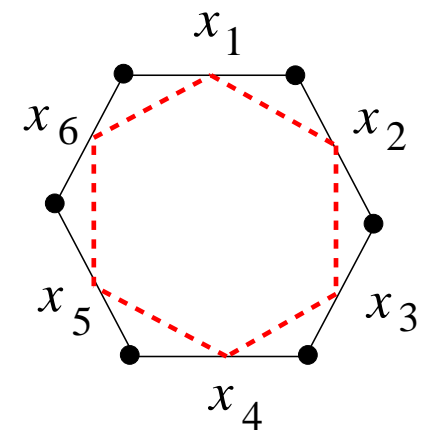

Figure 3: The convex hull of the centers of sides of the polygon $P_{6}$ is the dual polygon $* P_{6}$. A triangulation of the latter is an ideal triangulation producing a quiver.

we add a marked point inside of each of the two new sides of obtained polygons. See Fig 4. For example, cutting along an edge with the vertices labeled by points $x_{1}, x_{k}$, where $k \neq n+3,2$, we get a pair of polygons decorated by configurations of points

$$
\left(x_{1}^{-}, x_{2}, \ldots, x_{k-1}, x_{k}^{-}\right) \text {and }\left(x_{k}^{+}, x_{k+1}, \ldots, x_{n+3}, x_{1}^{+}\right) .
$$
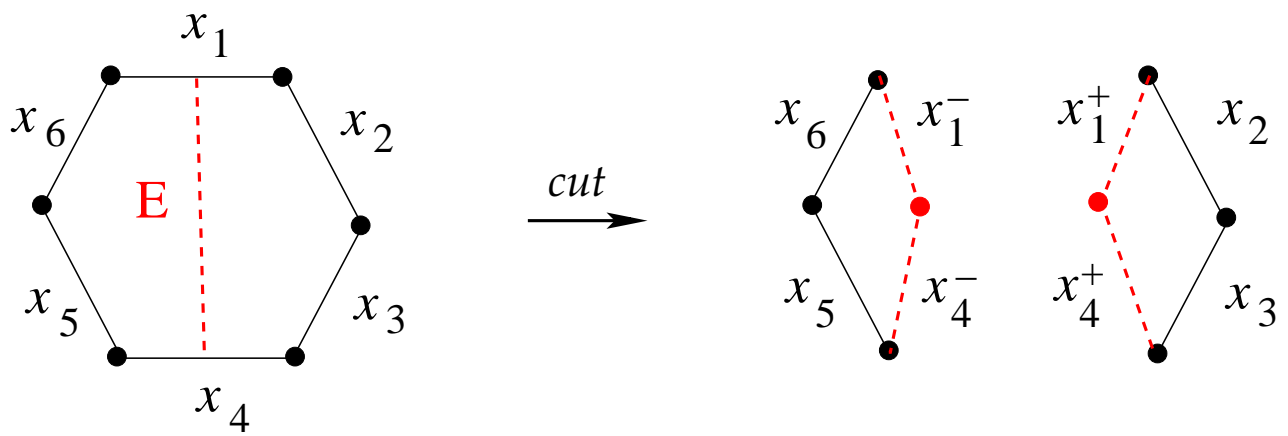

Figure 4: A boundary divisor of the cluster completion of $\mathcal{X}_{A_{3}}$ isomorphic to $\mathcal{X}_{A_{1}} \times \mathcal{X}_{A_{1}}$ corresponding to a cut of the hexagon by punctured segment, shown on the left.

For a general $\mathbb{S}$ we choose a non-boundary edge $E$ of an ideal triangulation $T$ of $\mathbb{S}$. Let $\mathbb{S}_{E}$ be the decorated surface obtained by cutting the surface along the edge $E$. We denote by $E_{-}$ and $E_{+}$the pair of boundary segments on $\mathbb{S}_{E}$ which glue back to $E$. The midpoints of $E_{-}$and $E_{+}$are new marked points. The rest of the marked points are inherited from $\mathbb{S}$. So the number of marked points always increases by two.

There is a canonical fibration with one dimensional fibers:

$$
\pi_{E}: \mathcal{X}_{P G L_{2}, \mathbb{S}} \longrightarrow \mathcal{X}_{P G L_{2}, \mathbb{S}_{E}}
$$

Namely, given a framed local system $(\mathcal{L}, \beta)$ on $\mathbb{S}$, we restrict the $\mathcal{L}$ to $\mathbb{S}_{E}$, getting a local system $\mathcal{L}_{E}$. Then the framing $\beta$ on $\mathcal{L}$ induces a framing $\beta_{E}$ on $\mathcal{L}_{E}$.

The fibers of fibration (11) can be parametrised, although non-canonically, as follows, see Fig 5. Let $\left(\mathcal{L}_{E}, \beta_{E}\right)$ be a framed $P G L_{2}$-local system on $\mathbb{S}_{E}$. Choose additional flat sections $y_{ \pm}$ of the $\mathbb{P}^{1}$-bundle near the new marked points over the boundary intervals $E_{ \pm}$. Assume that 
they differ from the ones provided by the framing $\beta_{E}$ on the left and on the right. Then there is a unique framed local system on $\mathbb{S}$ obtained from $\left(\mathcal{L}_{E}, \beta_{E}\right)$ by gluing $E_{-}$to $E_{+}$so that the section $y_{-}$is identified with $y_{+}$. Keeping $y_{-}$fixed and changing $y_{+}$we get the fiber. When $y_{+}$ approaches to the flat section at the end of $E_{+}$, we get the limiting framed local system on $\mathbb{S}_{E}$. He we use the orientation of the surface to tell which end.
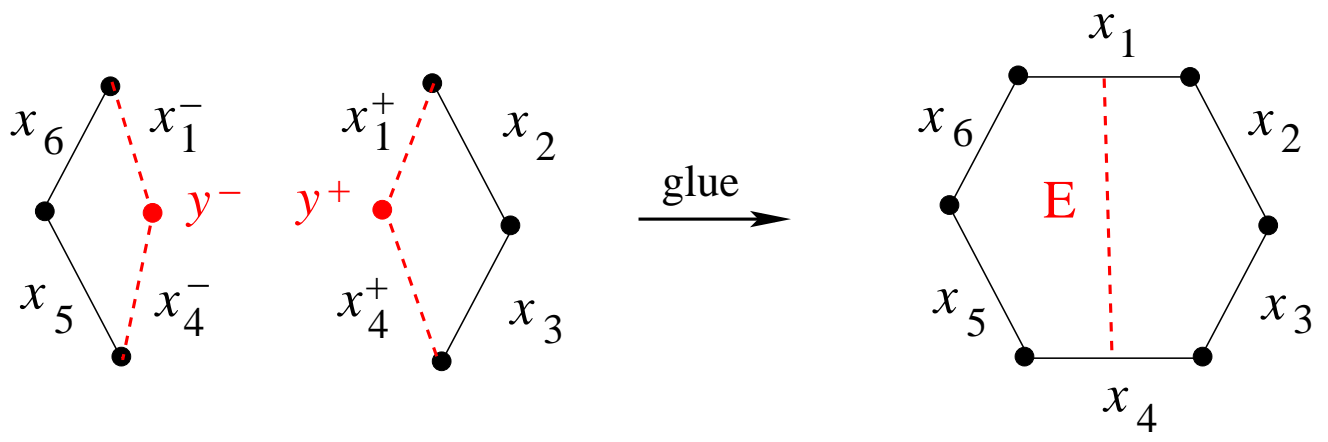

Figure 5: We add points $y^{-}$and $y^{+}$at the red vertices on the left, and glue the two configurations of points in a unique way so that the triples $\left(x_{1}^{-}, y^{-}, x_{4}^{-}\right)$and $\left(x_{1}^{+}, y^{+}, x_{4}^{+}\right)$are identified. After that we discard the $y$-points. Varying one of them, say $y^{+}$, we get the fibers of projection (11).

Special cones in the tropical space are parametrised by collections $\left\{E_{1}, \ldots, E_{k}\right\}$ of pairwise non-intersecting ideal non-boundary edges on $\mathbb{S}$. By the general construction, such a collection gives rise to a codimension $k$ stratum of the special completion of $\mathcal{X}_{P G L_{2}, \mathbb{S}}$. It is described as follows. Let $\mathbb{S}_{E_{1}, \ldots, E_{k}}$ be the surface obtained by cutting $\mathbb{S}$ along the edges $E_{1}, \ldots, E_{k}$. The vertices of the edges $E_{1}, \ldots, E_{k}$ become special points on the cutted surface.

Lemma 2.8. The stratum assigned to the collection $\left\{E_{1}, \ldots, E_{k}\right\}$ is naturally identified with the moduli spaces of framed $P G L_{2}$-local systems on $\mathbb{S}_{E_{1}, \ldots, E_{k}}$.

Proof. Let $T$ be an ideal triangulation of $\mathbb{S}$. Denote by $E d(T)$ the set of non-boundary edges of $T$. Then $E d(T)-\left\{E_{1}, \ldots, E_{k}\right\}$ is the set of non-boundary edges of the ideal triangulation of $\mathbb{S}_{E_{1}, \ldots, E_{k}}$ provided by $T$. Let $X_{E}^{T}$ be the cluster coordinate for the triangulation $T$ assigned to a non-boundary edge $E$ of $T$. Then the boundary stratum assigned to the collection $\left\{E_{1}, \ldots, E_{k}\right\}$ is obtained by letting the coordinates $X_{E}^{T}$, where $E \in\left\{E_{1}, \ldots, E_{k}\right\}$, to be zero.

Cluster Poisson variety $\mathcal{X}_{A_{n}}$, the Stasheff polytope and the Stasheff divisor. The $n$-dimensional Stasheff polytope is a convex polytope whose vertices are parametrised by complete triangulations of a convex polygon $P_{n+3}$. The $k$-dimensional faces are parametrised by triangulations missing $k$ diagonals. Here is a geometric realization of the Stascheff polytope.

A dihedral order of points $\left(x_{1}, \ldots, x_{n+3}\right)$ on the real projective line provides a connected component $\mathcal{M}_{0, n+3}^{0}(\mathbb{R})$ of the moduli space $\mathcal{M}_{0, n+3}(\mathbb{R})$, parametrising configurations of points on $\mathbb{P}^{1}(\mathbb{R})$ whose cyclic order is compatible with an orientation of $\mathbb{P}^{1}(\mathbb{R})$. It is nothing else but the Teichmuller space assigned to a disc with $n+3$ marked points on the boundary, discussed in Section 1.1. Its closure is identified with the Stasheff polytope.

The Zariski closure of the boundary of $\mathcal{M}_{0, n+3}^{0}(\mathbb{R})$ is the Stasheff divisor $B_{n}$ in $\overline{\mathcal{M}}_{0, n+3}$ [GM]. Its components intersect the Stasheff polytope by real codimension one faces. 
To clarify the structure of the Stasheff divisor, recall some basics about $\overline{\mathcal{M}}_{0, n+3}$. The complement $\overline{\mathcal{M}}_{0, n+3}-\mathcal{M}_{0, n+3}$ is a normal crossing divisor whose components are called the boundary divisors. They are parametrised by decompositions

$$
\left\{x_{1}, \ldots, x_{n+3}\right\}=I \cup J, \quad|I|,|J|>1 .
$$

Recall that we assign the points $x_{1}, \ldots, x_{n+3}$ to the vertices $p_{1}, \ldots, p_{n+3}$ of the convex polygon $P_{n+3}$.

The boundary divisor $D_{I, J}$, see (12), belongs to the Stasheff divisor if and only if the convex hull of the vertices from the set $I$ does not intersect the one for the set $J$. So the faces of the Stasheff divisor are products of the moduli spaces $\overline{\mathcal{M}}_{0, m}$. Their structure match the combinatorial structure of the Stasheff polytope.

Examples. 1. The cross-ratio (11) provides isomorphisms

$$
\mathcal{M}_{0,4} \stackrel{\sim}{\longrightarrow} \mathbb{P}^{1}-\{\infty,-1,0\}, \quad B_{1} \stackrel{\sim}{\longrightarrow}\{0, \infty\}, \quad \overline{\mathcal{M}}_{0,4} \stackrel{\sim}{\longrightarrow} \mathbb{P}^{1} .
$$

2. The complement $\overline{\mathcal{M}}_{0,5}-\mathcal{M}_{0,5}$ is a union of 10 projective lines. A choice of dihedral order of the points $\left(x_{1}, \ldots, x_{5}\right)$ provides a splitting of them into two pentagons. The Stasheff divisor $B_{2}$ is one of them. See $G$ for a more elaborate discussion of this example.

We summarise this discussion in the following Proposition.

Proposition 2.9. i) The space of real positive points $\mathcal{X}_{A_{n}}\left(\mathbb{R}_{>0}\right)$ is isomorphic to $\mathcal{M}_{0, n+3}^{0}(\mathbb{R})$.

ii) The space of the real positive points of $\widehat{\mathcal{X}}_{A_{n}}$ is isomorphic to the Stasheff polytope.

iii) The cluster modular group of $\mathcal{X}_{A_{n}}$ is identified with $\mathbb{Z} /(n+3) \mathbb{Z}$ if $n>1$ and $\mathbb{Z} / 2 \mathbb{Z}$ if $n=1$. Its generator acts on $\overline{\mathcal{M}}_{0, n+3}$ as the cyclic shift

$$
\left(x_{1}, x_{2}, \ldots, x_{n+3}\right) \longmapsto\left(x_{2}, \ldots, x_{n+3}, x_{1}\right) .
$$

Similarly, there is a scheme $\widehat{\mathcal{X}}_{\Delta}$ of finite type over $\mathbb{Z}$, the special completion of the cluster Poisson variety related to a root system $\Delta$. Recall the generalized Stasheff polytope $S_{\Delta}$ assigned to a root system $\Delta$ [FZII].

Proposition 2.10. The generalized Stasheff polytope $S_{\Delta}$ is a combinatorial skeleton of the scheme $\widehat{\mathcal{X}}_{\Delta}$. Precisely, $\widehat{\mathcal{X}}_{\Delta}$ has a stratification whose codimension $k$ strata match the codimension $k$ faces of the polytope $S_{\Delta}$ : The latter are products of the generalized Stasheff polytopes $S_{\Delta^{\prime}}$, and the former are products of the corresponding schemes $\widehat{\mathcal{X}}_{\Delta^{\prime}}$. One has $\widehat{\mathcal{X}}_{\Delta}\left(\mathbb{R}_{>0}\right)=S_{\Delta}$.

\subsection{Convexity in the framework of positive spaces.}

Convex subsets of the space of tropical points. Let $\mathcal{X}$ be a positive space. We define convex subsets of the space of the tropical points $\mathcal{X}\left(\mathbb{A}^{t}\right)$, where $\mathbb{A}$ is either $\mathbb{Z}$, or $\mathbb{Q}$, or $\mathbb{R}$.

Let $V$ be a real vector space. A convex domain in $V$ is an intersection, possibly infinite, of half spaces given by the inequalities $l(x) \leq c$ where $l(x)$ are linear functions on $V$, and $c$ are constants. We look at this as follows. A split torus $\mathrm{T}$ is the simplest basic example of a positive space. The space $\mathrm{T}\left(\mathbb{Z}^{t}\right)$ of its integral tropical points is a free abelian group given by the characters of $T$. One has $T\left(\mathbb{Q}^{t}\right)=T\left(\mathbb{Z}^{t}\right) \otimes \mathbb{Q}$. Linear functions with integral coefficients 
are nothing else but the tropicalizations of the positive regular functions on the torus $\mathrm{T}$. We generalise this by replacing the torus $\mathrm{T}$ by an arbitrary positive space $\mathcal{X}$.

Denote by $\mathbb{L}_{+}(\mathcal{X})$ the semiring of positive regular functions on $\mathcal{X}$. It is the set of rational functions which are Laurent polynomials with positive integral coefficients in every coordinate system of the atlas. Denote by $\mathbb{E}(\mathcal{X})$ the set of extremal elements of the cone $\mathbb{L}_{+}(\mathcal{X})$, i.e. the elements which can not be presented as a sum of two non-zero elements of the cone.

A function $E \in \mathbb{E}(\mathcal{X})$ and a constant $c_{E} \in \mathbb{Q} \cup \infty$ give rise to a basic convex subset

$$
C\left(E, c_{E}\right)=\left\{x \in \mathcal{X}\left(\mathbb{Q}^{t}\right) \mid E^{t}(x) \leq c_{E}\right\} .
$$

Convex subsets of $\mathcal{X}\left(\mathbb{Q}^{t}\right)$ are defined as intersections of such subsets. If $c_{E}=\infty$, the corresponding subset is $\mathcal{X}\left(\mathbb{Q}^{t}\right)$. So if all but finitely many $c_{E}$ 's are infinite, we get a finite intersection of basic convex subsets. We extend the addition in $\mathbb{Q}$ to an addition in $\mathbb{Q} \cup \infty$ by setting $a+\infty=\infty$. One defines similarly convex subsets of $\mathcal{X}\left(\mathbb{A}^{t}\right)$ :

Definition 2.11. i) $A$ convex subset $A \subset \mathcal{X}\left(\mathbb{A}^{t}\right)$ is defined by inequalities

$$
A:=\left\{x \in \mathcal{X}\left(\mathbb{A}^{t}\right) \mid E^{t}(x) \leq a_{E} \text { for each } E \in \mathbb{E}(\mathcal{X}), \quad a_{E} \in \mathbb{A} \cup \infty\right\} .
$$

ii) Given $n$ convex subsets

$$
A_{i}=\left\{x \in \mathcal{X}\left(\mathbb{A}^{t}\right) \mid E^{t}(x) \leq a_{E}^{(i)} \text { for each } E \in \mathbb{E}(\mathcal{X})\right\},
$$

their Minkowski sum $A_{1} * \ldots * A_{n}$ is defined by

$$
A_{1} * \ldots * A_{n}:=\left\{x \in \mathcal{X}\left(\mathbb{A}^{t}\right) \mid E^{t}(x) \leq a_{E}^{(1)}+\ldots+a_{E}^{(n)} \text { for each } E \in \mathbb{E}(\mathcal{X})\right\} .
$$

Each coordinate system identifies the set $\mathcal{X}\left(\mathbb{A}^{t}\right)$ with $\mathbb{A}^{\operatorname{dim} \mathcal{X}}$. Our convex subsets are identified with certain convex (in the usual sense) subsets of $\mathbb{A}^{\operatorname{dim} \mathcal{X}}$.

Spherical convex subsets. We start again with a motivation. Let $V$ be a rational vector space and $\mathbb{S}(V)$ the sphere of the rays in $V$. Convex domains in $\mathbb{S}(V)$ are projectivisation of convex cones in $V$. A convex cone in $V$ is an intersection of half spaces defined by inequalities $l(v) \leq 0$, where $l$ are linear functions on $V$. We conclude that the semiring of positive integral Laurent polynomials determines the classical convex structure of the sphere $\mathbb{S}(V)$. The real spherical tropical space for a split torus $\mathrm{T}$ is nothing else but the sphere $\mathbb{S}(V)$. This suggests the following generalization.

Definition 2.12. Let $\mathcal{X}$ be a positive space.

i) Convex subsets of the tropical space $\mathcal{S} \mathcal{X}\left(\mathbb{A}^{t}\right)$ are the subsets

$$
S_{F}=\left\{x \in \mathcal{S} \mathcal{X}\left(\mathbb{Q}^{t}\right) \mid F^{t}(x) \leq 0\right\}, \quad F \in \mathbb{L}_{+}(\mathcal{X}) .
$$

ii) The Minkowski sum of convex subsets $S_{F_{1}} * S_{F_{2}}$ is given by

$$
S_{F_{1}} * S_{F_{2}}:=S_{F_{1} F_{2}} .
$$


Given two functions $F_{1}, F_{2} \in \mathbb{L}_{+}(\mathcal{X})$, we have

$$
S_{F_{1}+F_{2}}=S_{F_{1}} \cap S_{F_{2}}
$$

Thus finite intersections of the convex subsets in $\mathcal{X}\left(\mathbb{Q}^{t}\right)$ are convex subsets. This leads to

Lemma 2.13. The spherical convex subsets form a semiring with the addition given by the intersection of the subsets, and the multiplication given by the Minkowski sum. The map $F \longrightarrow$ $S_{F}$ is a morphism of the semiring $\mathbb{L}_{+}(\mathcal{X})$ to the semiring of convex subsets of $\mathcal{X}$.

Finally, we can allow infinite intersections of the basic spherical convex subsets.

\section{References}

[FG1] Fock V.V., Goncharov A.B. Moduli spaces of local systems and higher Teichmuller theory. Publ. Math. IHES, n. 103 (2006) 1-212. ArXiv math.AG/0311149.

[FG2] Fock V.V., Goncharov A.B.: Cluster ensembles, quantization and the dilogarithm. Ann. Sci. École Norm. Sup. vol 42, (2009) 865-929. ArXiv math.AG/0311245.

[FG3] Fock V.V., Goncharov A.B. Dual Teichmüller and lamination spaces. Handbook of Teichmüller theory. Vol. I, 647-684, IRMA Lect. Math. Theor. Phys., 11, Eur. Math. Soc., Zürich, 2007.ArXive math.DG/0510312.

[FZ] Fomin S., Zelevinsky A.: Cluster algebras: Notes for the CDM-03 conference. arXiv:math/0311493.

[FZI] Fomin S., Zelevinsky A.: Cluster algebras. I. J. Amer. Math. Soc. 15 (2002), no. 2, $497-529$

[FZII] Fomin S., Zelevinsky A.: Cluster algebras. II. Inv. Math., 154 (2003), no. 1, 63-121.

[FZL] Fomin S., Zelevinsky A.: The Laurent phenomenon. Adv. in Appl. Math. 28 (2002), no. 2, 119-144.

[G] Goncharov A.B.: Pentagon relation for the quantum dilogarithm and quantized $\mathcal{M}_{0,5}^{\mathrm{cyc}}$. Progress in Math. Vol 265 (2007) Birkhauser Verlag, Basel, 415-428.

[GM] Goncharov A.B., Manin Yu.I.: Multiple zeta-motives and moduli spaces $M_{0, n}$. Compositio Math, 140 (2004), 1-14. arXiv:math/0204102.

[L] Lusztig G.: Total positivity in reductive groups, in Lie theory and geometry: In honor of Bertram Kostant, Progress in Mathematics 123, Birkhauser, 1994, 531-568.

[M] Masur H.: The extension of the Weil-Petersson metric to the boundary of Teichmuller space. Duke Math. J. Volume 43, Number 3 (1976), 623-635.

[SW] Speyer D., Williams L.: The tropical totally positive Grassmannian arXiv:math/0312297. 